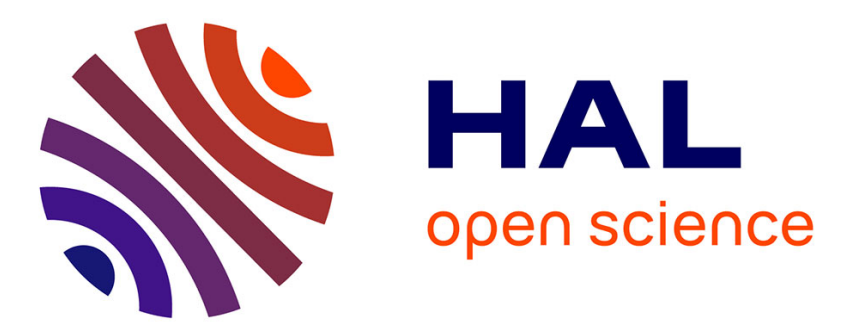

\title{
Exposition professionnelle au formaldéhyde en France en 2013
}

\author{
Loïc Garras, Laurène Delabre, Marie Houot, Corinne Pilorget
}

\section{To cite this version:}

Loïc Garras, Laurène Delabre, Marie Houot, Corinne Pilorget. Exposition professionnelle au formaldéhyde en France en 2013. 35e congrès national de médecine et santé au travail, Jun 2018, PARIS, France. p. 396, 10.1016/j.admp.2018.03.418 . hal-02266070

\section{HAL Id: hal-02266070 https://hal.science/hal-02266070}

Submitted on 26 Aug 2019

HAL is a multi-disciplinary open access archive for the deposit and dissemination of scientific research documents, whether they are published or not. The documents may come from teaching and research institutions in France or abroad, or from public or private research centers.
L'archive ouverte pluridisciplinaire HAL, est destinée au dépôt et à la diffusion de documents scientifiques de niveau recherche, publiés ou non, émanant des établissements d'enseignement et de recherche français ou étrangers, des laboratoires publics ou privés. 


\title{
Exposition professionnelle au formaldéhyde en France en 2013
}

\author{
LoïcGarras, LaurèneDelabre, MarieHouot, CorinnePilorget
}

Objectifs

Le formaldéhyde est utilisé dans de nombreux secteurs pour ses différentes propriétés depuis de nombreuses années. Il a été classé «cancérogène avéré pour l’homme » par le Centre international de recherche sur le cancer en 2004 et « cancérogène supposé pour l'être humain » par l'Union européenne en 2014. L'objectif de ce travail est de décrire l'exposition professionnelle au formaldéhyde en France en 2013 et d'identifier les secteurs et professions les plus exposants.

\section{Méthodes}

Dans le cadre du programme Matgéné, une matrice emplois-expositions (MEE) au formaldéhyde a été conçue par expertise. Cette MEE attribue pour chacun des emplois exposés (profession dans un secteur d'activité) entre 1950 et 2017 des indices d'exposition (probabilité, intensité, fréquence). Le croisement de cette MEE avec les données du recensement de la population de 2013 a permis d'estimer la prévalence d'exposition professionnelle au formaldéhyde en France selon la profession et le secteur d'activité.

\section{Résultats}

En 2013, 290000 travailleurs étaient exposés professionnellement au formaldéhyde en France, soit $1,3 \%$ de la population au travail. Les secteurs d'activité dont la prévalence est la plus élevée sont les secteurs de l'habillement (18,4 \% d'exposés), la fabrication de textile (18,2 \%) et l'industrie du cuir et de la chaussure (12,8\%). Par ailleurs, les secteurs présentant le plus grand nombre d'exposés sont les activités pour la santé humaine (environ 81000 exposés), la fabrication de produits métalliques (29 000) et les autres services personnels comprenant les services funéraires et les manucures (28 700). Les ouvriers qualifiés de type artisanal et les artisans du textile, de l'habillement, et du cuir et les ouvriers qualifiés de type industriel du travail des métaux ont les prévalences d'exposition les plus élevées (respectivement $35 \%$, 35 \% et $30 \%$ ). Les professions avec le plus grand nombre de travailleurs exposés sont les ouvriers qualifiés de type industriel du travail des métaux (40 200), les aides-soignants et professions assimilées (32 900) et les techniciens médicaux (24 000).

\section{Conclusions}

Ces résultats sont les seuls à ce jour qui documentent l'exposition au formaldéhyde en France pour l'ensemble des travailleurs quel que soit leur statut (salariés ou indépendants). Ils permettent d'identifier les professions et les secteurs d'activité contribuant le plus à l'exposition au formaldéhyde (prévalences les plus élevées) mais également les professions et secteur dans lesquels le nombre de personnes exposées est le plus important. Ces informations sont utiles pour la surveillance de ce risque professionnel et la priorisation des actions de prévention à mettre en place. 